\title{
Statistical analysis of the precision of the Match method
}

\author{
R. Lehmann, P. von der Gathen, M. Rex, and M. Streibel \\ Alfred Wegener Institute for Polar and Marine Res., Res. Unit Potsdam, Telegrafenberg A43, D-14473 Potsdam, Germany
}

Received: 2 February 2005 - Published in Atmos. Chem. Phys. Discuss.: 24 May 2005

Revised: 9 September 2005 - Accepted: 23 September 2005 - Published: 18 October 2005

\begin{abstract}
The Match method quantifies chemical ozone loss in the polar stratosphere. The basic idea consists in calculating the forward trajectory of an air parcel that has been probed by an ozone measurement (e.g., by an ozonesonde or satellite instrument) and finding a second ozone measurement close to this trajectory. Such an event is called a "match". A rate of chemical ozone destruction can be obtained by a statistical analysis of several tens of such match events. Information on the uncertainty of the calculated rate can be inferred from the scatter of the ozone mixing ratio difference (second measurement minus first measurement) associated with individual matches. A standard analysis would assume that the errors of these differences are statistically independent. However, this assumption may be violated because different matches can share a common ozone measurement, so that the errors associated with these match events become statistically dependent. Taking this effect into account, we present an analysis of the uncertainty of the final Match result. It has been applied to Match data from the Arctic winters 1995, 1996, 2000, and 2003. For these ozonesonde Match studies the effect of the error correlation on the uncertainty estimates is rather small: compared to a standard error analysis, the uncertainty estimates increase by $15 \%$ on average. However, the effect may be more pronounced for typical satellite Match analyses: for an Antarctic satellite Match study (2003), the uncertainty estimates increase by $60 \%$ on average.

The analysis showed that the random errors of the ozone measurements and the "net match errors", which result from a displacement of the second ozone measurement of a match from the required position, are of similar magnitude. This demonstrates that the criteria for accepting a match (maximum trajectory duration, match radius, spread of trajectory clusters etc.) ensure that, given the unavoidable ozone-
\end{abstract}

Correspondence to: R. Lehmann

(rlehmann@awi-potsdam.de) measurement errors, the magnitude of the net match errors is adequate. The estimate of the random errors of the ozonesonde measurements agrees well with laboratory results.

\section{Introduction}

The Match method was developed to quantify chemical ozone loss in the Arctic stratosphere (von der Gathen et al., 1995; Rex et al., 1998, 1999). The basic idea is the following: After an air parcel has been probed by an ozonesonde, its forward trajectory is calculated. If a second ozonesonde comes close to this trajectory, i.e. its distance from the trajectory is smaller than a pre-defined threshold, then the measurements of the two ozonesondes form a "match". This can happen by chance or may be attained by launching the second sonde intentionally so that it approaches the trajectory at the appropriate time. In the final analysis, backward trajectories may also be applied to determine matches. It is possible that an ozone measurement forms matches with more than one other ozone measurement.

A variant of the original Match method uses satellite data instead of ozonesonde measurements (Sasano et al., 2000; Terao et al., 2002; and Sect. 9 of the present paper). Although all formulae derived in the present paper are also applicable to satellite or other data, we will prefer the terminology of the original Match method (e.g., "first sonde" and "second sonde" of a match).

Under ideal circumstances (no measurement errors, no trajectory error, zero distance of the second sonde from the trajectory), the difference of the ozone mixing ratios measured by the first and second sonde would be equal to the change of the ozone mixing ratio along the trajectory. Under the assumption that mixing can be neglected, this is equal to the chemical ozone loss in the corresponding air parcel. 


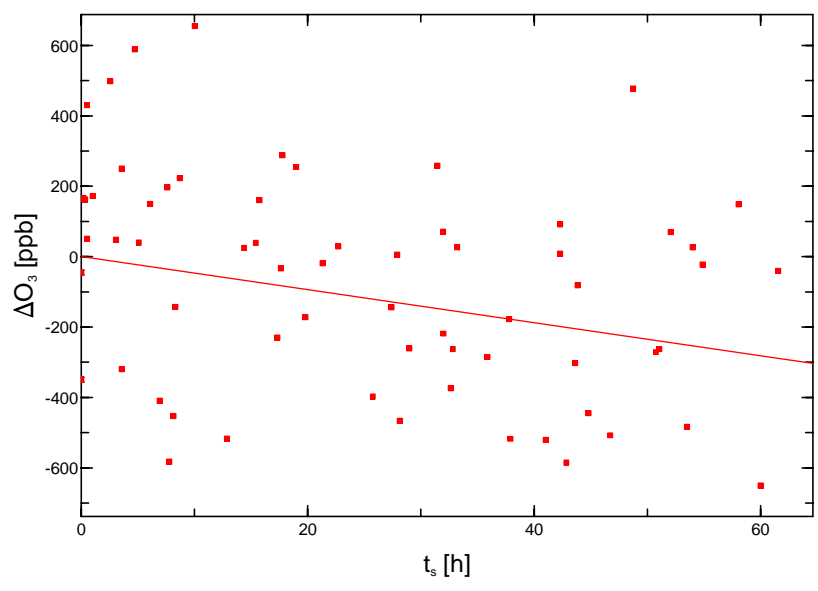

Fig. 1. Difference $\Delta \mathrm{O}_{3}$ of the ozone mixing ratio determined by the second and first sonde of matches in dependence on the time $t_{S}$ that the corresponding trajectory spent in sunlight. The slope of the regression line is the mean ozone loss rate, expressed as ozone loss per sunlit time. The data represent all Arctic match events of 14-28 January 1995, $475 \mathrm{~K}$.

As the above-listed errors are non-zero in reality, it is not possible to draw conclusions from a single pair of ozone observations. However, if several tens of such pairs are available, then an ozone loss rate can be obtained by statistical methods. For this, the differences between the ozone mixing ratio of the first and second measurement of several pairs of "matching" sondes are plotted versus a variable that is expected to correlate with ozone destruction, usually the time that the corresponding trajectories spent in sunlight. Then a linear fit, which is forced through the origin $(0,0)$ of the coordinate system, is performed. The slope of that line yields an estimate of the mean ozone loss rate, e.g., ozone loss per hour of sunlight. An example is given in Fig. 1.

A standard assumption of a linear regression analysis is the statistical independence of the errors of the quantities entering the analysis. In the case of the Match method, these quantities are the ozone mixing ratio differences of several matches. As one ozone measurement may enter several matches, the errors of those matches will not be uncorrelated. The influence of this effect on the Match analysis will be investigated in this paper. It turns out that the linear regression still yields an unbiased estimate of the mean ozone loss rate (Sect. 5), but the calculation of the corresponding uncertainty ("error bars") is affected.

First, the uncertainties associated with the Match method are reviewed in Sect. 2. Then the effect of the use of correlated ozone data on the uncertainty of the Match results is illustrated with the help of a highly simplified example in Sect. 3. The formulae for the exact numerical treatment of this effect are derived in Sects. 4-6 and summarised in algorithmic form in Sect. 7. Finally, results of the application of the new formulae to data from five Match campaigns are presented in Sects. 8 and 9.
The method presented involves a separate estimation of the random measurement errors of the ozonesondes and the random errors introduced by the Match method itself (ozone deviation due to trajectory errors and non-zero distance of the second sonde from the trajectory). It turns out that the errors of these two categories are of similar magnitude. This balance of errors demonstrates that the criteria for accepting a match (maximum trajectory duration, match radius, spread of trajectory clusters etc.) were suitably selected. The estimate of the random measurement errors of the ozonesondes agrees well with laboratory results (Sect. 8.2).

\section{Uncertainties associated with the Match method}

\subsection{Random errors versus systematic errors}

In general, measurement errors can be classified as systematic ("accuracy") or random errors ("precision").

Systematic errors are inherent in the measurement technique and can only be estimated by an investigation of this technique itself. Systematic errors of the Match method can be caused, e.g., by approximations in the code that calculates the vertical position of trajectories. For a discussion of systematic errors see Rex et al. (1998), Grooß and Müller (2003), and Morris et al. (2005).

Random errors can be estimated by statistical methods from the scatter of measurements. The errors of the Match method contain a significant random component, arising, e.g., from random errors of the ozone measurements and of the meteorological data that enter the trajectory calculations. Information on the magnitude of the random errors associated with individual match events can be obtained from the scatter of the corresponding ozone mixing ratio differences around the regression line describing the mean ozone loss (cf. Fig. 1). Then straight-forward statistical methods can provide an estimate of the random error of the slope of the regression line itself, i.e. of the ozone loss rate determined by the Match method.

This paper will deal exclusively with random errors, i.e. with the precision of the Match method. The term "uncertainty" will be used in this sense.

Precision may be quantified by the standard deviation of the random variable describing the quantity of interest. This standard deviation is usually unknown, but an estimate may be obtained from realisations of the random variable. Error bars are a graphical representation of the precision, in the above-cited Match publications they represent one standard deviation. Similarly, here we will use the term "precision estimate", or alternatively "error bar", for denoting an estimate of the standard deviation of the random errors of the Match results. 


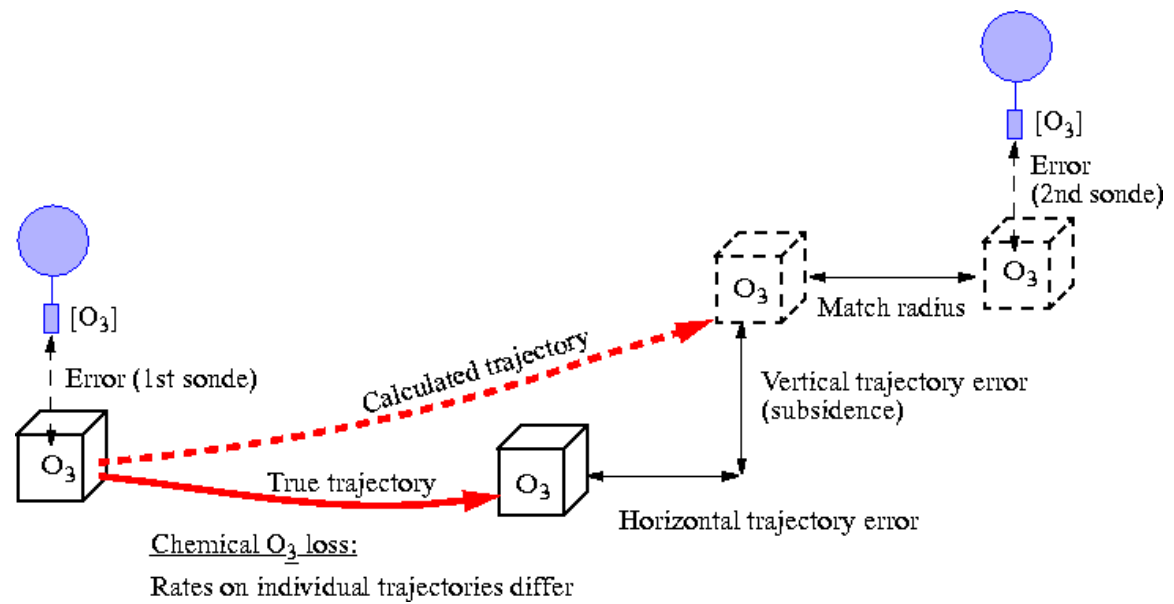

Fig. 2. Schematic representation of the uncertainties associated with the Match method.

2.2 Sources of uncertainties associated with the Match method

The uncertainties associated with the Match method have the following sources, which are schematically depicted in Fig. 2:

a) measurement error of the first and second ozonesonde of a match; this includes the error of the ozone measurement itself and the errors of the pressure and temperature measurements, which are translated into an error of the potential temperature level to which an ozone measurement is assigned; furthermore, the ozone mixing ratio at the point of the sonde measurement may deviate from the mean mixing ratio in a larger air parcel (of the scale resolved by the trajectory calculations) due to small-scale ozone variations;

b) trajectory error (horizontal and vertical), which results in an ozone measurement displaced from the required position;

c) non-zero match radius, i.e. the distance of the measurement of the second sonde from the trajectory at the time of the measurement; this also results in an ozone measurement displaced from the required position;

d) deviation of the ozone loss rate on an individual trajectory from the mean loss rate in a region of interest.

The Match method attempts to limit the above-listed error sources if possible (Rex et al., 1998, 1999):

a) Standard operation procedures for participating ozonesonde stations have been worked out. However, certain measurement errors are inevitable, unless improved ozonesondes become available. b) The Match method includes procedures to limit the effect of the trajectory error: A cluster of trajectories around the trajectory of interest is calculated. If these trajectories diverge significantly, it is assumed that the main trajectory is more error-prone. In this case it is discarded, i.e. not used for establishing a match. In order to ensure that vertical trajectory errors are not translated into large errors of the ozone mixing ratio, only ozone profiles with vertical gradients below a certain threshold are used.

c) In order to limit the error of the ozone mixing ratio resulting from a non-zero match radius, a suitable maximum match radius is applied.

d) The variability of the ozone loss rate on different trajectories is determined by the inhomogeneity of the chlorine activation in the region of interest and also by differences of the solar zenith angle during solar illumination along the trajectories. It is thus objectively present, but it may, to some degree, be influenced by the choice of the region of interest (whole polar vortex, vortex core etc.).

Error a) is an instrumental error, whereas errors b)-d) are related to the technique of Match. We will denote the combined effect of b)-d) as "net match error" ("net", because the measurement errors a) are not included).

The exact knowledge of the sources of errors will not be of relevance for the derivations in the subsequent sections. However, this work will be based on the fact that there are two categories of random errors: those associated with the ozone measurements, which may be shared by several matches, and individual net errors of each match. All of these errors will be assumed to be statistically independent from each other. This assumption needs some discussion: Two match events having a common first sonde also 

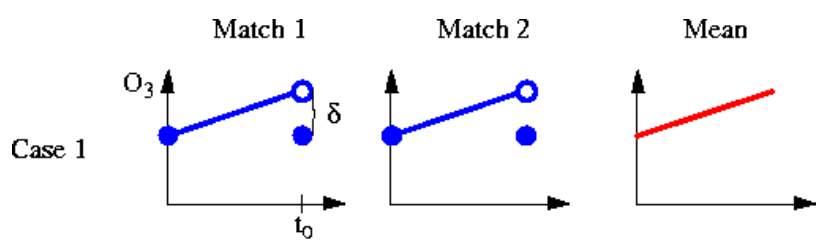

Case 2
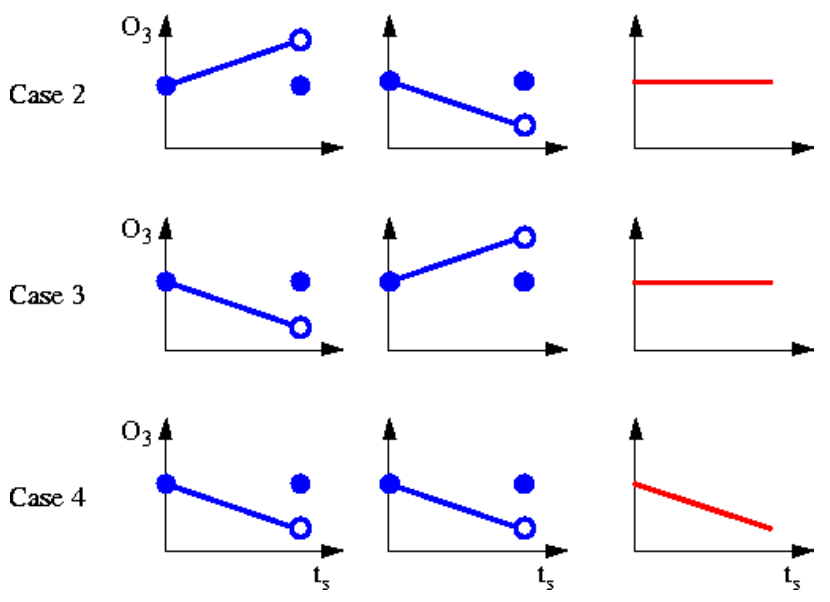

Fig. 3. Illustration of the uncertainty of the slope of the linear regression line in the case of two independent matches. Full circles denote the ozone mixing ratio at the position of the first and second sonde of the corresponding match. Empty circles indicate the ozone mixing ratio obtained by sonde measurements, having an error $\pm \delta$. The ozone loss rate derived from the measurements is represented by the slope of the straight line in each panel. $t_{0}$ denotes the time $t_{S}$ that the corresponding trajectory spent in sunlight.

share a common trajectory segment, starting at the position of the first sonde measurement. That is why their trajectory errors can be correlated, which may lead to correlated net match errors. If this effect was significant, then it would be reflected in the covariance (of the ozone mixing ratio difference) of pairs of matches sharing a common first sonde. This covariance would have a larger absolute value than the corresponding covariance for pairs of matches that share a common ozonesonde, but no common trajectory segment, i.e. for the case "second sonde of first match = first sonde of second match". However, for the ozonesonde data analysed in Sect. 8, this was not the case, so that we can conclude that the correlation between the net match errors is negligible.

\section{Illustration of the effect of correlated matches}

As mentioned in Sects. 1 and 2, the ozone mixing ratio differences (second measurement minus first measurement) associated with individual match events, like in Fig. 1, may be correlated, because one ozone measurement may be part of several match events. This will influence the estimation of the precision of the slope of the regression line (cf. Fig. 1), i.e. of the ozone loss rate. In order to illustrate this effect,
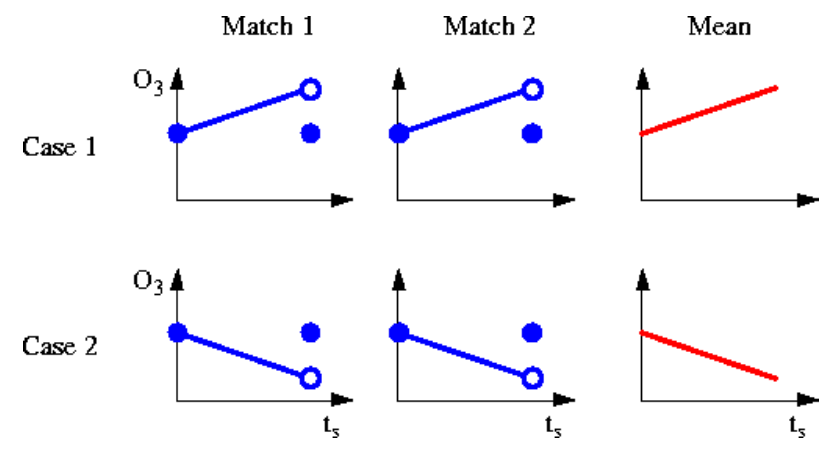

Fig. 4. Illustration of the uncertainty of the slope of the linear regression line in the case of two matches having a common second sonde. Symbols are as in Fig. 3.

we consider a highly simplified example: We assume that only two matches occurred and that there was no ozone loss along the corresponding two trajectories. Furthermore, the sunlit times associated with the two matches are assumed to be equal and will be denoted by $t_{0}$. Then the estimate of the ozone loss rate determined by the linear regression is equal to the mean of the ozone loss rates calculated for the individual match events. In order to simplify the situation further, we assume that only one ozone measurement per match has a measurement error and that this error can only assume two values $(+\delta$ and $-\delta)$ of equal probability. All other errors associated with the Match method are assumed to vanish. Now we consider three cases:

a) The two matches are independent: If we assume that the measurements of the second sondes of these matches are associated with an error, then the ozone mixing ratio differences measured in the two match events, $d_{1}$ and $d_{2}$, may assume the following values (with equal probabilities):

$$
\begin{array}{ll}
d_{1}=+\delta, & d_{2}=+\delta \text { or } \\
d_{1}=+\delta, & d_{2}=-\delta \text { or } \\
d_{1}=-\delta, & d_{2}=+\delta \text { or } \\
d_{1}=-\delta, & d_{2}=-\delta .
\end{array}
$$

The calculated mean ozone loss rate will be $\frac{\delta}{t_{0}}$ in the first case, 0 in the second and third case, and $-\frac{\delta}{t_{0}}$ in the fourth case. This is illustrated in Fig. 3.

b) The two matches have a common second sonde: If we assume that this sonde is associated with an error, then the ozone mixing ratio differences measured may assume the following values (with equal probabilities):

$$
\begin{aligned}
& d_{1}=+\delta, \quad d_{2}=+\delta \text { or } \\
& d_{1}=-\delta, \quad d_{2}=-\delta
\end{aligned}
$$




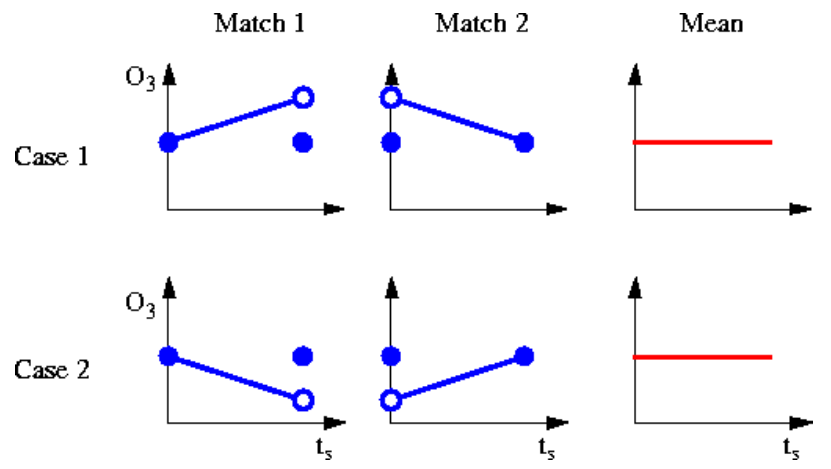

Fig. 5. Illustration of the uncertainty of the slope of the linear regression line in the case of two matches sharing one sonde that is first sonde for one of the matches and second sonde for the other match. Symbols are as in Fig. 3.

The calculated mean ozone loss rate will be $\frac{\delta}{t_{0}}$ in the first case, and $-\frac{\delta}{t_{0}}$ in the second case. This is illustrated in Fig. 4. As the extreme values of the ozone destruction rate $\left(\frac{\delta}{t_{0}}\right.$ and $\left.-\frac{\delta}{t_{0}}\right)$ of case 1 still occur, but the middle value (0) does not appear, the variance of the estimated ozone loss rate has increased compared to case 1 .

c) The two matches have a common sonde, which is second sonde for the first match and first sonde for the second match: If we assume that this sonde is associated with an error, then the ozone mixing ratio differences measured may assume the following values (with equal probabilities):

$d_{1}=+\delta, d_{2}=-\delta$ or

$d_{1}=-\delta, d_{2}=+\delta$.

The calculated mean ozone loss rate will be 0 in both cases. This is illustrated in Fig. 5. In this example the variance of the ozone loss rate is zero, i.e. clearly smaller than in the first case.

The results of these three very simple examples are summarised in Fig. 6. They illustrate that pairs of matches with a common second (or first) sonde will increase the variance of the estimated ozone loss rate, compared to the case of independent matches. Pairs of matches with a common sonde, that is first sonde for one of the matches and second sonde for the other one, will decrease the variance of the estimated ozone loss rate. These simple considerations will be confirmed by the calculations in Sect. 6 .

\section{Statistical description of the uncertainties associated with the Match method}

As a basis for estimating the uncertainty of the final Match result, we are going to provide a statistical description of the
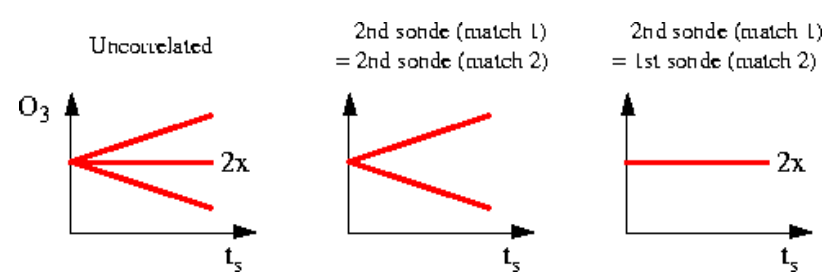

Fig. 6. Illustration of the uncertainty of the slope of the linear regression line for the three cases displayed in Figs. 3-5.

uncertainties discussed in Sect. 2. First we introduce the following notations:

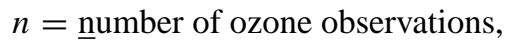

$m=$ number of match events,

$\bar{r}=$ mean ozone loss rate (loss per sunlit time) for the atmospheric region probed by the matches under consideration $[\mathrm{ppb} / \mathrm{h}]$ (to be estimated),

$c_{k}=$ ozone mixing ratio determined by the $k$-th sonde measurement, $k=1, \ldots, n$,

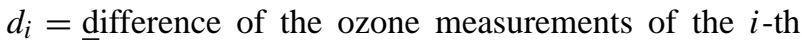
match, $i=1, \ldots, m$,

$t_{i}=$ sunlit time of the $i$-th match, $i=1, \ldots, m$,

$\delta_{k}=$ error of the $k$-th ozone measurement, $k=1, \ldots, n$,

$\Delta_{i}=$ net match error of the $i$-th match event, resulting from the combined effect of the trajectory error, the non-zero match radius, and the deviation of the ozone loss rate on the $i$-th trajectory from the mean ozone loss rate $\bar{r}, i=1, \ldots, m$,

$\epsilon_{i}=$ total error associated with the $i$-th match, $i=1, \ldots, m$,

$k_{1}(i)=$ index of the first sonde measurement of the $i$-th match, $i=1, \ldots, m$,

$k_{2}(i)=$ index of the second sonde measurement of the $i$-th match, $i=1, \ldots, m$.

In order to simplify the notation, we define the following vectors:

$\boldsymbol{c}=\left(c_{1}, \ldots, c_{n}\right)^{T}, \boldsymbol{d}=\left(d_{1}, \ldots, d_{m}\right)^{T}, \boldsymbol{t}=\left(t_{1}, \ldots, t_{m}\right)^{T}$,

$\boldsymbol{\delta}=\left(\delta_{1}, \ldots, \delta_{n}\right)^{T}, \boldsymbol{\Delta}=\left(\Delta_{1}, \ldots, \Delta_{m}\right)^{T}, \quad \boldsymbol{\epsilon}=\left(\epsilon_{1}, \ldots, \epsilon_{m}\right)^{T}$,

where ${ }^{T}$ denotes the transpose of a vector (or matrix).

For storing the information about which sonde measurement contributes to which match events, we define the following $m \times n$ matrix $\mathbf{M}$ ("Match matrix"), each row of which corresponds to a match event and each column of which corresponds to an ozone observation:

$m_{i k}=\left\{\begin{aligned} 1 & \text { if the second sonde of the } i \text {-th match is } \\ & \text { sonde } k, \\ -1 & \text { if the first sonde of the } i \text {-th match is sonde } k, \\ 0 & \text { if the } i \text {-th match does not use sonde } k .\end{aligned}\right.$ 
For example, the matrix describing a first match between the ozone observations number 1 and 2 and a second match between the ozone observations number 2 and 3 is

$\mathbf{M}=\left(\begin{array}{ccc}-1 & 1 & 0 \\ 0 & -1 & 1\end{array}\right)$.

The ozone mixing ratio difference $d_{i}$ associated with the $i$-th match event is the difference between the two ozone mixing ratios obtained by the second and by the first sonde measurement of this match event:

$d_{i}=c_{k_{2}(i)}-c_{k_{1}(i)}$

or, in matrix form,

$\boldsymbol{d}=\mathbf{M} \cdot \boldsymbol{c}$.

The ozone mixing ratio difference $d_{i}$ deviates from the ozone loss $\bar{r} \cdot t_{i}$, expected from the mean loss rate, by the total match error $\epsilon_{i}$ :

$d_{i}=\bar{r} \cdot t_{i}+\epsilon_{i}$

The total match error $\epsilon_{i}$ may be expressed by the errors of the ozonesonde measurements and the net match error of the $i$-th match as follows:

$\epsilon_{i}=\delta_{k_{2}(i)}-\delta_{k_{1}(i)}+\Delta_{i}$.

Taking into account the definition of the Match matrix $\mathbf{M}$, we may write Eqs. (3) and (4) in vector form:

$\boldsymbol{d}=\bar{r} \cdot \boldsymbol{t}+\boldsymbol{\epsilon}$,

$\boldsymbol{\epsilon}=\mathbf{M} \cdot \boldsymbol{\delta}+\boldsymbol{\Delta}$.

We assume that the errors $\delta_{k}$ of all individual ozone observations and the net match errors $\Delta_{i}$ are unbiased, i.e. do not comprise a systematic error:

$\mathrm{E}(\boldsymbol{\delta})=\mathbf{0}$,

$\mathrm{E}(\boldsymbol{\Delta})=\mathbf{0}$,

where $E($.$) denotes the expected value of a random vector,$ and $\mathbf{0}$ is the null vector of appropriate size. From these two equations and Eq. (6) it follows that the total match errors are also unbiased:

$\mathrm{E}(\boldsymbol{\epsilon})=\mathbf{0}$.

We assume that the errors $\delta_{k}$ of all individual ozone observations are statistically independent and their variances are identical, namely $\sigma_{\delta}^{2}$. Analogously, the net match errors $\Delta_{i}$ are assumed to be statistically independent of each other and independent of the measurement errors $\delta_{k}$ (cf. Sect. 2.2); their variance, which is assumed to be independent of $i$, is denoted by $\sigma_{\Delta}^{2}$. Then the corresponding covariance matrices can be written as:

$$
\begin{gathered}
\operatorname{Cov}(\boldsymbol{\delta})=\sigma_{\delta}^{2} \cdot \mathbf{I}, \\
\operatorname{Cov}(\boldsymbol{\Delta})=\sigma_{\Delta}^{2} \cdot \mathbf{I},
\end{gathered}
$$

where the matrices $\mathbf{I}$ are identity matrices of appropriate size $(n \times n$ or $m \times m)$. From these two equations and the statistical independence of the errors $\delta_{k}, k=1, \ldots, n$, and $\Delta_{i}$, $i=1, \ldots, m$, we can obtain an expression for the covariance matrix of the total match errors:

$$
\begin{aligned}
\operatorname{Cov}(\boldsymbol{\epsilon})= & \mathbf{M} \cdot \operatorname{Cov}(\boldsymbol{\delta}) \cdot \mathbf{M}^{T}+\operatorname{Cov}(\boldsymbol{\Delta}), \\
& \quad \text { because of Eq. (6), } \\
= & \mathbf{M} \cdot \sigma_{\delta}^{2} \cdot \mathbf{I} \cdot \mathbf{M}^{T}+\sigma_{\Delta}^{2} \cdot \mathbf{I}, \\
& \quad \text { because of Eqs. (10) and (11), } \\
= & \sigma_{\delta}^{2} \cdot \mathbf{M} \cdot \mathbf{M}^{T}+\sigma_{\Delta}^{2} \cdot \mathbf{I} .
\end{aligned}
$$

The elements $\mu_{i j}$ of the matrix $\mathbf{M} \cdot \mathbf{M}^{T}$ are:

$\mu_{i j}=\left\{\begin{aligned} 2 & \text { if } i=j, \\ 1 & \text { if the matches } i \text { and } j(j \neq i) \text { have a common } \\ & \text { first or a common second sonde, } \\ -1 & \text { if the second sonde of match } i \text { is the first sonde } \\ & \text { of match } j(j \neq i) \text { or vice versa, } \\ 0 \text { else. } & \end{aligned}\right.$

For the example in Eq. (1) we obtain

$\mathbf{M} \cdot \mathbf{M}^{T}=\left(\begin{array}{cc}2 & -1 \\ -1 & 2\end{array}\right)$

In order to show the effect of the off-diagonal elements of $\mathbf{M} \cdot \mathbf{M}^{T}$ more clearly, we split this matrix into two matrices containing the diagonal and the off-diagonal elements of $\mathbf{M} \cdot \mathbf{M}^{T}$, respectively:

$\mathbf{M} \cdot \mathbf{M}^{T}=2 \cdot \mathbf{I}+\boldsymbol{\Omega}$,

where the elements $\omega_{i j}$ of the matrix $\boldsymbol{\Omega}$ are:

$\omega_{i j}=\left\{\begin{aligned} 1 & \text { if the matches } i \text { and } j(j \neq i) \text { have a common } \\ & \text { first or a common second sonde, } \\ -1 & \text { if the second sonde of match } i \text { is the first sonde } \\ & \text { of match } j(j \neq i) \text { or vice versa, } \\ 0 & \text { else. }\end{aligned}\right.$

From this definition it is evident that $\boldsymbol{\Omega}$ is symmetric. Replacing $\mathbf{M} \cdot \mathbf{M}^{T}$ in Eq. (12) by the expression in Eq. (14), we obtain:

$\operatorname{Cov}(\boldsymbol{\epsilon})=\left(\sigma_{\Delta}^{2}+2 \sigma_{\delta}^{2}\right) \cdot \mathbf{I}+\sigma_{\delta}^{2} \cdot \boldsymbol{\Omega}$

After defining

$\sigma^{2}=\sigma_{\Delta}^{2}+2 \sigma_{\delta}^{2}$

we can write Eq. (16) in the form

$\operatorname{Cov}(\boldsymbol{\epsilon})=\sigma^{2} \cdot \mathbf{I}+\sigma_{\delta}^{2} \cdot \boldsymbol{\Omega}$ 


\section{The linear regression for calculating the ozone loss rate}

In order to obtain an estimate $\hat{r}$ of the mean ozone loss rate $\bar{r}$, a linear regression of the ozone mixing ratio differences $d_{i}$ on the corresponding sunlit times $t_{i}$ is performed. The fit is forced to pass through the origin $(0,0)$ of the coordinate system, because for a sunlit-time interval of zero length no ozone destruction is expected to occur. This linear regression analysis determines the value of $r$ that minimizes the expression

$\sum_{i=1}^{m}\left(d_{i}-r \cdot t_{i}\right)^{2}=(\boldsymbol{d}-r \cdot \boldsymbol{t})^{T}(\boldsymbol{d}-r \cdot \boldsymbol{t})$.

The solution $\hat{r}$ of this minimization problem is

$\hat{r}=\frac{\sum_{i=1}^{m} t_{i} \cdot d_{i}}{\sum_{i=1}^{m} t_{i}^{2}}=\frac{1}{\boldsymbol{t}^{T} \boldsymbol{t}} \cdot \boldsymbol{t}^{T} \boldsymbol{d}$.

Together with Eq. (5) this yields

$$
\begin{aligned}
\hat{r} & =\frac{1}{\boldsymbol{t}^{T} \boldsymbol{t}} \cdot \boldsymbol{t}^{T} \cdot(\bar{r} \cdot \boldsymbol{t}+\boldsymbol{\epsilon}) \\
& =\frac{1}{\boldsymbol{t}^{T} \boldsymbol{t}} \cdot \boldsymbol{t}^{T} \boldsymbol{t} \cdot \bar{r}+\frac{1}{\boldsymbol{t}^{T} \boldsymbol{t}} \cdot \boldsymbol{t}^{T} \boldsymbol{\epsilon} \\
& =\bar{r}+\frac{1}{\boldsymbol{t}^{T} \boldsymbol{t}} \cdot \boldsymbol{t}^{T} \boldsymbol{\epsilon} .
\end{aligned}
$$

This proves that the estimate $\hat{r}$ is unbiased, because $\mathrm{E}(\boldsymbol{\epsilon})=\mathbf{0}$ according to Eq. (9). Furthermore, it leads to the following equation for the variance of the estimate $\hat{r}$ :

$$
\begin{aligned}
\sigma_{\hat{r}}^{2} & =\left(\frac{1}{\boldsymbol{t}^{T} \boldsymbol{t}} \cdot \boldsymbol{t}^{T}\right) \cdot \operatorname{Cov}(\boldsymbol{\epsilon}) \cdot\left(\frac{1}{\boldsymbol{t}^{T} \boldsymbol{t}} \cdot \boldsymbol{t}^{T}\right)^{T} \\
& =\left(\frac{1}{\boldsymbol{t}^{T} \boldsymbol{t}} \cdot \boldsymbol{t}^{T}\right) \cdot\left(\sigma^{2} \cdot \mathbf{I}+\sigma_{\delta}^{2} \cdot \boldsymbol{\Omega}\right) \cdot\left(\frac{1}{\boldsymbol{t}^{T} \boldsymbol{t}} \cdot \boldsymbol{t}^{T}\right)^{T},
\end{aligned}
$$

because of Eq. (18),

$$
=\frac{1}{\boldsymbol{t}^{T} \boldsymbol{t}} \cdot \sigma^{2}+\frac{\boldsymbol{t}^{T} \boldsymbol{\Omega} \boldsymbol{t}}{\left(\boldsymbol{t}^{T} \boldsymbol{t}\right)^{2}} \cdot \sigma_{\delta}^{2} .
$$

After defining

$\omega=\frac{\boldsymbol{t}^{T} \boldsymbol{\Omega} \boldsymbol{t}}{\boldsymbol{t}^{T} \boldsymbol{t}}=\frac{\sum_{i j} \omega_{i j} \cdot t_{i} \cdot t_{j}}{\sum_{i=1}^{m} t_{i}^{2}}$,

we can rewrite Eq. (22) in the form

$\sigma_{\hat{r}}^{2}=\frac{1}{\boldsymbol{t}^{T} \boldsymbol{t}} \cdot\left(\sigma^{2}+\omega \cdot \sigma_{\delta}^{2}\right)$.

\section{Estimation of uncertainties}

The variances $\sigma^{2}=\sigma_{\Delta}^{2}+2 \cdot \sigma_{\delta}^{2}$ and $\sigma_{\delta}^{2}$ in Eq. (24) are unknown. In this section we are going to derive estimates for them.

\subsection{Information on $\sigma^{2}$}

Taking into account that the diagonal elements of $\boldsymbol{\Omega}$ are zero, we see from Eq. (18) that the diagonal elements of $\operatorname{Cov}(\boldsymbol{\epsilon})$ are $\sigma^{2}$, i.e. together with Eq. (9) we obtain

$\mathrm{E}\left(\epsilon_{i}^{2}\right)=\sigma^{2}$,

and thus, because of Eq. (3),

$\mathrm{E}\left\{\left(d_{i}-\bar{r} \cdot t_{i}\right)^{2}\right\}=\sigma^{2}$.

This means that $\left(d_{i}-\bar{r} \cdot t_{i}\right)^{2}, \quad i=1, \ldots, m$, are unbiased estimates of $\sigma^{2}$. Consequently, the expression $\frac{1}{m} \cdot \sum_{i=1}^{m}\left(d_{i}-\bar{r} \cdot t_{i}\right)^{2}$ is also an unbiased estimate of $\sigma^{2}$, but with a smaller variance than the individual terms. As $\bar{r}$ is unknown, we are going to replace it by the estimate $\hat{r}$. This has the consequence that the arising estimate for $\sigma^{2}$ will no longer be unbiased. However, it should still contain much information on $\sigma^{2}$. That is why we consider the following sum and calculate its expected value:

$s_{1}=\sum_{i=1}^{m}\left(d_{i}-\hat{r} \cdot t_{i}\right)^{2}$.

The sum $s_{1}$ is also known as "chi-square" $\left(\chi^{2}\right)$. After recalling the definition of $\omega$ in Eq. (23), we can obtain the following expression for the expected value of $s_{1}$ (see Appendix B):

$\mathrm{E}\left(s_{1}\right)=(m-1) \cdot \sigma^{2}-\omega \cdot \sigma_{\delta}^{2}$.

For the data analysed in Sects. 8.1-8.3, the mean values of $m-1$ and $\omega$ are 41.3 and 1.1, respectively. This means that $\mathrm{E}\left(s_{1}\right)$ is dominated by the term containing $\sigma^{2}$.

6.2 Special case: $\sigma_{\delta}=0$

If $\sigma_{\delta}=0$, i.e. if the sonde measurement errors vanish and thus the (total) match errors are uncorrelated, then Eq. (28) reduces to

$\mathrm{E}\left(s_{1}\right)=(m-1) \cdot \sigma^{2}$.

This means that an unbiased estimate $\hat{s}^{2}$ of $\sigma^{2}$ can be obtained from

$\hat{s}^{2}=\frac{s_{1}}{m-1}$.

Substituting this value for $\sigma^{2}$ in Eq. (24), we obtain the "classical" error estimate

$\hat{s}_{\hat{r}}^{2}=\frac{1}{\boldsymbol{t}^{T} \boldsymbol{t}} \cdot \frac{s_{1}}{m-1}=\frac{1}{m-1} \cdot \frac{\sum_{i=1}^{m}\left(d_{i}-\hat{r} \cdot t_{i}\right)^{2}}{\sum_{i=1}^{m} t_{i}^{2}}$. 


\subsection{Special case: $\sigma_{\Delta}=0$}

If $\sigma_{\Delta}=0$, i.e. if the net match errors vanish, then $\sigma^{2}=2 \cdot \sigma_{\delta}^{2}$, and Eq. (28) reduces to

$\mathrm{E}\left(s_{1}\right)=\{2 \cdot(m-1)-\omega\} \cdot \sigma_{\delta}^{2}$.

Thus an unbiased estimate $\hat{s}_{\delta}^{2}$ of $\sigma_{\delta}^{2}$ can be obtained from

$\hat{s}_{\delta}^{2}=\frac{s_{1}}{2 \cdot(m-1)-\omega}$.

Substituting this value for $\sigma_{\delta}^{2}$ in Eq. (24), we obtain the error estimate

$$
\begin{aligned}
\hat{s}_{\hat{r}}^{2} & =\frac{1}{\boldsymbol{t}^{T} \boldsymbol{t}} \cdot(2+\omega) \cdot \frac{s_{1}}{2 \cdot(m-1)-\omega} \\
& =\frac{1+\frac{\omega}{2}}{m-1-\frac{\omega}{2}} \cdot \frac{\sum_{i=1}^{m}\left(d_{i}-\hat{r} \cdot t_{i}\right)^{2}}{\sum_{i=1}^{m} t_{i}^{2}},
\end{aligned}
$$

This is identical to Eq. (31) if $\omega=0$, e.g. if the covariance matrix $\operatorname{Cov}(\boldsymbol{\epsilon})$ is diagonal and hence the match errors are uncorrelated.

For the derivation of Eq. (34) the effect of the correlation between the ozone data used in different match events, due to the multiple use of the same ozone measurements in several matches, has been taken into account. As the estimate $\hat{s}_{\hat{r}}^{2}$ in Eq. (34) has been derived under the assumption $\sigma_{\Delta}^{2}=0$, i.e. the ozone measurement errors $\delta_{k}$ alone determine the total match error, it may provide an upper bound of the effect of taking into account the above-mentioned correlation. For the data analysed in Sects. 8.1-8.3, the error bar $\hat{s}_{\hat{r}}$ from Eq. (34) is, on average, $24 \%$ larger than the "classical" estimate according to Eq. (31).

In the case of vanishing net match errors, it is easy to see from Eq. (34) how the precision estimate for the final Match result is affected by the multiple use of ozone measurements in several matches: Pairs of matches $i$ and $j, i \neq j$, that share a common first or a common second sonde measurement correspond to $\omega_{i j}=1$ (cf. Eq. 15) and thus yield a positive summand in $\omega$ (cf. Eq. 23). According to Eq. (34) they thus contribute to an increase of the error estimate $\hat{s}_{\hat{r}}^{2}$. The reverse is true for pairs of matches $i$ and $j, i \neq j$, for which the second sonde measurement of match $i$ coincides with the first sonde measurement of match $j$ (or vice versa) and consequently $\omega_{i j}=-1$ according to Eq. (15). This confirms the conclusions from the highly simplified example in Sect. 3.

\subsection{Information on $\sigma_{\delta}^{2}$}

If we compare the off-diagonal elements in Eq. (18), we obtain for $i \neq j$ :

$\mathrm{E}\left(\epsilon_{i} \cdot \epsilon_{j}\right)=\omega_{i j} \cdot \sigma_{\delta}^{2}$,

and thus, because of Eq. (3),

$\mathrm{E}\left\{\left(d_{i}-\bar{r} \cdot t_{i}\right) \cdot\left(d_{j}-\bar{r} \cdot t_{j}\right)\right\}=\omega_{i j} \cdot \sigma_{\delta}^{2}$.
If $\omega_{i j} \neq 0$, then $\omega_{i j}=1$ or $\omega_{i j}=-1$ and consequently $\omega_{i j}^{2}=1$, so that we obtain from Eq. (36) by multiplication by $\omega_{i j}$ :

$\mathrm{E}\left\{\omega_{i j} \cdot\left(d_{i}-\bar{r} \cdot t_{i}\right) \cdot\left(d_{j}-\bar{r} \cdot t_{j}\right)\right\}=\sigma_{\delta}^{2}$.

Now we proceed as in Sect. 6.1: Eq. (37) means that $\omega_{i j} \cdot\left(d_{i}-\bar{r} \cdot t_{i}\right) \cdot\left(d_{j}-\bar{r} \cdot t_{j}\right)$, with $i, j$ such that $\omega_{i j} \neq 0$, $i, j=1, \ldots, m$, are unbiased estimates of $\sigma_{\delta}^{2}$. Consequently, the arithmetic mean of these expressions is also an unbiased estimate of $\sigma_{\delta}^{2}$, but with a smaller variance than the individual terms. As $\bar{r}$ is unknown, we are going to replace it by the estimate $\hat{r}$. This has the consequence that the arising estimate for $\sigma_{\delta}^{2}$ will no longer be unbiased. However, it should still contain much information on $\sigma_{\delta}^{2}$. That is why we consider the following sum and calculate its expected value:

$s_{2}=\sum_{i=1}^{m} \sum_{j=1\left(\omega_{i j} \neq 0\right)}^{m} \omega_{i j} \cdot\left(d_{i}-\hat{r} \cdot t_{i}\right) \cdot\left(d_{j}-\hat{r} \cdot t_{j}\right)$.

As adding the zero terms corresponding to $\omega_{i j}=0$ does not alter this sum, we can write $s_{2}$ also in the form

$s_{2}=\sum_{i=1}^{m} \sum_{j=1}^{m} \omega_{i j} \cdot\left(d_{i}-\hat{r} \cdot t_{i}\right) \cdot\left(d_{j}-\hat{r} \cdot t_{j}\right)$.

After defining

$\omega_{1}=\sum_{i=1}^{m} \sum_{j=1}^{m} \omega_{i j}^{2}$,

$\omega_{2}=\frac{(\boldsymbol{\Omega} \cdot \boldsymbol{t})^{T} \cdot(\boldsymbol{\Omega} \cdot \boldsymbol{t})}{\boldsymbol{t}^{T} \boldsymbol{t}}$,

we can obtain the following expression for the expected value of $s_{2}$ (see Appendix C):

$\mathrm{E}\left(s_{2}\right)=-\omega \cdot \sigma^{2}+\left(\omega_{1}-2 \cdot \omega_{2}+\omega^{2}\right) \cdot \sigma_{\delta}^{2}$.

As $\omega_{i j}=0$ or $\omega_{i j}= \pm 1, i, j=1, \ldots, m$, the value $\omega_{1}$ is equal to the number of non-zero elements of the matrix $\boldsymbol{\Omega}$. For the data analysed in Sects. 8.1-8.3, the mean values of $\omega_{1}, \omega_{2}$, and $\omega$, are $179,5.5$, and 1.1 , respectively. This means that $\mathrm{E}\left(s_{2}\right)$ is dominated by the term containing $\sigma_{\delta}^{2}$.

\subsection{Estimates for $\sigma^{2}$ and $\sigma_{\delta}^{2}$}

If we define the $2 \times 2$ matrix $\mathbf{A}$ by

$\mathbf{A}=\left(\begin{array}{cc}m-1 & -\omega \\ -\omega & \omega_{1}-2 \cdot \omega_{2}+\omega^{2}\end{array}\right)$,

then Eqs. (28) and (42) may be written as

$\mathrm{E}\left(\begin{array}{l}s_{1} \\ s_{2}\end{array}\right)=\mathbf{A} \cdot\left(\begin{array}{c}\sigma^{2} \\ \sigma_{\delta}^{2}\end{array}\right)$.

Consequently,

$\mathrm{E}\left\{\mathbf{A}^{-1} \cdot\left(\begin{array}{l}s_{1} \\ s_{2}\end{array}\right)\right\}=\mathbf{A}^{-1} \cdot \mathbf{A} \cdot\left(\begin{array}{c}\sigma^{2} \\ \sigma_{\delta}^{2}\end{array}\right)=\left(\begin{array}{c}\sigma^{2} \\ \sigma_{\delta}^{2}\end{array}\right)$. 
This means that $\hat{s}^{2}$ and $\hat{s}_{\delta}^{2}$ given by

$$
\left(\begin{array}{c}
\hat{s}^{2} \\
\hat{s}_{\delta}^{2}
\end{array}\right)=\mathbf{A}^{-1} \cdot\left(\begin{array}{l}
s_{1} \\
s_{2}
\end{array}\right)
$$

are unbiased estimates of $\sigma^{2}$ and $\sigma_{\delta}^{2}$, respectively. The inverse of the matrix $\mathbf{A}$ is

$\mathbf{A}^{-1}=\frac{1}{D} \cdot\left(\begin{array}{cc}\omega_{1}-2 \cdot \omega_{2}+\omega^{2} & \omega \\ \omega & m-1\end{array}\right)$

with

$$
\begin{aligned}
D & =(m-1) \cdot\left(\omega_{1}-2 \cdot \omega_{2}+\omega^{2}\right)-\omega^{2} \\
& =(m-1) \cdot\left(\omega_{1}-2 \cdot \omega_{2}\right)+(m-2) \cdot \omega^{2} .
\end{aligned}
$$

Thus we finally obtain from Eq. (46):

$\hat{s}^{2}=\frac{1}{D} \cdot\left\{\left(\omega_{1}-2 \cdot \omega_{2}+\omega^{2}\right) \cdot s_{1}+\omega \cdot s_{2}\right\}$,

$\hat{s}_{\delta}^{2}=\frac{1}{D} \cdot\left\{\omega \cdot s_{1}+(m-1) \cdot s_{2}\right\}$.

\subsection{Estimate for $\sigma_{\hat{r}}^{2}$}

If we substitute the estimates $\hat{s}^{2}$ for $\sigma^{2}$ and $\hat{s}_{\delta}^{2}$ for $\sigma_{\delta}^{2}$ from Eqs. (49), (50) in Eq. (24), then we obtain the desired estimate $\hat{s}_{\hat{r}}^{2}$ of $\sigma_{\hat{r}}^{2}$ :

$$
\begin{aligned}
& \hat{s}_{\hat{r}}^{2}= \frac{1}{\boldsymbol{t}^{T} \boldsymbol{t}} \cdot\left(\hat{s}^{2}+\omega \cdot \hat{s}_{\delta}^{2}\right) \\
&=\frac{1}{\boldsymbol{t}^{T} \boldsymbol{t}} \cdot \frac{1}{D} \cdot\left\{\left(\omega_{1}-2 \cdot \omega_{2}+\omega^{2}\right) \cdot s_{1}+\omega \cdot s_{2}\right. \\
&\left.\quad+\omega^{2} \cdot s_{1}+(m-1) \cdot \omega \cdot s_{2}\right\} \\
&=\frac{1}{\boldsymbol{t}^{T} \boldsymbol{t}} \cdot \frac{1}{D} \cdot\left\{\left(\omega_{1}-2 \cdot \omega_{2}+2 \cdot \omega^{2}\right) \cdot s_{1}+m \cdot \omega \cdot s_{2}\right\} .
\end{aligned}
$$

It might be desirable to calculate the standard deviation of this estimate, i.e. the "error bars of the error bars", and investigate whether it is a minimum-variance estimate. However, this remains beyond the scope of the present paper.

\subsection{Avoidance of unfeasible values}

It cannot be excluded that the estimate $\hat{s}_{\delta}^{2}$ of the variance $\sigma_{\delta}^{2}$ becomes negative. As a negative variance is unrealistic, this case will be treated by assuming $\sigma_{\delta}^{2}=0$ and applying Eq. (31). If on the other hand, $\hat{s}_{\delta}^{2}>\hat{s}^{2}$, which corresponds to a negative estimate for the variance $\sigma_{\Delta}^{2}$, then it will be assumed that $\sigma_{\Delta}^{2}=0$ and Eq. (34) will be applied. These modifications might, in principle, destroy the unbiasedness of the final estimate $\hat{s}_{\hat{r}}^{2}$. However, for the 96 match ensembles investigated in Sect. 8, the introduction of the above-described sign-restrictions changed the mean value of the estimate $\hat{s}_{\hat{r}}^{2}$ by only $0.04 \%$.
In order to prevent a division by zero in Eqs. (49) and (50), the complete algorithm in the next section will check whether $D$ according to Eq. (48) is zero. If this is the case, then the "classical" error estimate Eq. (31) will be applied. This might occur, e.g., if $\boldsymbol{\Omega}=\mathbf{0}$, i.e. if all match events use independent sonde measurements, in which case the application of Eq. (31) yields the correct estimate. However, in the examples of Sects. 8 and 9 this never occurred.

\section{Complete formulae for estimating the precision of Match}

Summarising the findings of the previous sections, we obtain the following algorithm for the calculation of estimates for the precision of the Match results. For these estimates we will also use the more illustrative denotation "error bars".

Input:

$m=$ number of match events,

$d_{i}=$ difference of the ozone mixing ratio (second minus first sonde measurement) of the $i$-th match, $i=1, \ldots, m$,

$t_{i}=$ sunlit time of the $i$-th match, $i=1, \ldots, m$,

$k_{1}(i)=$ identifier (index or name) of the first sonde measurement of the $i$-th match, $i=1, \ldots, m$,

$k_{2}(i)=$ identifier of the second sonde measurement of the $i$-th match, $i=1, \ldots, m$.

Output:

$\hat{r}=$ estimated ozone loss rate,

$\hat{s}_{\hat{r}}^{2}=$ estimated variance of $\hat{r}$.

\section{Algorithm:}

1. Calculate an estimate of the ozone loss rate by linear regression:

$\hat{r}=\frac{\sum_{i=1}^{m} t_{i} \cdot d_{i}}{\sum_{i=1}^{m} t_{i}^{2}}$.

2. Set up the matrix $\boldsymbol{\Omega}$ that stores the information on ozonesonde measurements shared by two or more match events:

$\omega_{i j}=\left\{\begin{array}{c}1 \text { if } k_{1}(i)=k_{1}(j) \text { or } k_{2}(i)=k_{2}(j), \text { and } i \neq j, \\ -1 \text { if } k_{2}(i)=k_{1}(j) \text { or } k_{1}(i)=k_{2}(j), \text { and } i \neq j, \\ 0 \text { else. }\end{array}\right.$

3. Calculate the auxiliary expressions $s_{1}, s_{2}, \omega, \omega_{1}, \omega_{2}$, and $D$ :

$$
\begin{aligned}
& s_{1}=\sum_{i=1}^{m}\left(d_{i}-\hat{r} \cdot t_{i}\right)^{2}, \\
& s_{2}=\sum_{i=1}^{m} \sum_{j=1}^{m} \omega_{i j} \cdot\left(d_{i}-\hat{r} \cdot t_{i}\right) \cdot\left(d_{j}-\hat{r} \cdot t_{j}\right),
\end{aligned}
$$




$$
\begin{aligned}
\omega & =\frac{\sum_{i j} \omega_{i j} \cdot t_{i} \cdot t_{j}}{\sum_{i=1}^{m} t_{i}^{2}}, \\
\omega_{1} & =\sum_{i=1}^{m} \sum_{j=1}^{m} \omega_{i j}^{2}, \\
\omega_{2} & =\frac{(\boldsymbol{\Omega} \cdot \boldsymbol{t})^{T} \cdot(\boldsymbol{\Omega} \cdot \boldsymbol{t})}{\boldsymbol{t}^{T} \boldsymbol{t}}, \text { where } \boldsymbol{t}=\left(t_{1}, \ldots, t_{m}\right)^{T}, \\
D & =(m-1) \cdot\left(\omega_{1}-2 \cdot \omega_{2}\right)+(m-2) \cdot \omega^{2} .
\end{aligned}
$$

4. If $D=0$, then use the "classical" error bar: goto step 8 .

5. Calculate estimates $\hat{s}^{2}$ for $\sigma^{2}$ and $\hat{s}_{\delta}^{2}$ for $\sigma_{\delta}^{2}$ :

$\hat{s}^{2}=\frac{1}{D} \cdot\left\{\left(\omega_{1}-2 \cdot \omega_{2}+\omega^{2}\right) \cdot s_{1}+\omega \cdot s_{2}\right\}$,

$\hat{s}_{\delta}^{2}=\frac{1}{D} \cdot\left\{\omega \cdot s_{1}+(m-1) \cdot s_{2}\right\}$.

6. Sign check for $\hat{s}_{\delta}^{2}$ and $\hat{s}_{\Delta}^{2}=\hat{s}^{2}-2 \cdot \hat{s}_{\delta}^{2}$ :

If $\hat{s}_{\delta}^{2}<0$, then goto step 8 .

If $\hat{s}_{\delta}^{2}>\frac{1}{2} \cdot \hat{s}^{2}$, then goto step 9 .

7. Calculate error bar:

$\hat{s}_{\hat{r}}^{2}=\frac{1}{\sum_{i=1}^{m} t_{i}^{2}} \cdot\left(\hat{s}^{2}+\omega \cdot \hat{s}_{\delta}^{2}\right)$.

stop.

8. "Classical" error bar (zero sonde measurement errors):

$\hat{s}_{\hat{r}}^{2}=\frac{1}{m-1} \cdot \frac{\sum_{i=1}^{m}\left(d_{i}-\hat{r} \cdot t_{i}\right)^{2}}{\sum_{i=1}^{m} t_{i}^{2}}$.

stop.

9. Error bar for zero net match errors, i.e. only sonde measurement errors occur:

$\hat{s}_{\hat{r}}^{2}=\frac{1+\frac{\omega}{2}}{m-1-\frac{\omega}{2}} \cdot \frac{\sum_{i=1}^{m}\left(d_{i}-\hat{r} \cdot t_{i}\right)^{2}}{\sum_{i=1}^{m} t_{i}^{2}}$.

stop.

\section{Application to ozonesonde Match campaigns}

\subsection{Data}

The analysis in the following Sects. 8.2 and 8.3 is based on all data from the Arctic Match campaigns of the winters 1994/95 (potential temperature levels $450 \mathrm{~K}, 475 \mathrm{~K}, 500 \mathrm{~K}$; Rex et al., 1999), 1995/96 (475 K; Rex et al., 1997), 1999/2000 (450 K, 475 K, 500 K; Rex et al., 2002), and 2002/03 (475 K; Streibel et al., 2005). These data correspond to 96 match ensembles, i.e. the calculation of 96 ozone loss rates by the application of a linear regression like in Fig. 1.
8.2 Estimates of the measurement errors and the net match errors

For the data introduced in the previous subsection, the mean values of the estimates $\hat{s}_{\delta}^{2}$ and $\hat{s}_{\Delta}^{2}$ are $2.4 \cdot 10^{4} \mathrm{ppb}^{2}$ and $2.7 \cdot 10^{4} \mathrm{ppb}^{2}$, respectively. This means that the errors of the ozone measurements and the net match errors are of similar magnitude.

The net match errors are controlled by the criteria for accepting a pair of ozonesonde measurements as a match (cf. Sect. 2.2). If the net errors associated with the Match method were significantly larger than the errors of the ozonesonde measurements, this would indicate that the criteria for accepting a match are too tolerant. Then the information of the sonde measurements would not be exploited adequately, because the net match errors dominate the final result. If, on the other hand, the net match errors were significantly smaller than the sonde errors, then the criteria for accepting a match would be too restrictive. As a consequence the accepted number of matches would be unnecessarily small, which again leads to an inadequate exploitation of the information of the sonde measurements. The above-noticed balance of the net match errors and the sonde errors indicates that the criteria for accepting a match were suitably chosen.

Extracting the square root from the mean value of $\hat{s}_{\delta}^{2}$ yields $\hat{s}_{\delta}=156 \mathrm{ppb}$, which corresponds to $6 \%$ of the mean ozone mixing ratio (of all sonde measurements entering the mentioned matches). This is consistent with the precision of ozonesonde measurements of 5.7\%, obtained during the Jülich Ozone Sonde Intercomparison Experiment JOSIE (quadratic mean of the precision values for SPC-6A and ENSCI sondes with a $1 \% \mathrm{KI}$ solution in the height range of 15 $25 \mathrm{~km}$ given by Smit and Straeter, 2004a, Table 9, and Smit and Straeter, 2004b, Table 14).

\subsection{Comparison of new and old error bars}

Up to now the "classical" error bars, according to Eq. (61), have been used in the Match analysis. Figure 7 shows both the new error bars, according to $\hat{s}_{\hat{r}}$ in Eq. (60), and the old ones. It can be seen that the new error bars are slightly larger on average. For ozone loss rates greater than approximately $2 \mathrm{ppb} / \mathrm{h}$, the loss rates are greater than the old error bars, so that the ozone loss can be considered significant. This does not change when the new error bars are used instead of the old ones.

Figure 8 displays the ratio of the new error bars to the old ones. It varies between 0.96 and 1.68 . The $90 \%$-quantile is 1.32 , i.e. for $90 \%$ of the data points the ratio is less than 1.32 . The mean value of the ratio is 1.15 .

On average every ozonesonde measurement was used in slightly more than 2 match events (see triangular arrowhead in Fig. 8). In order to express statements like this more 


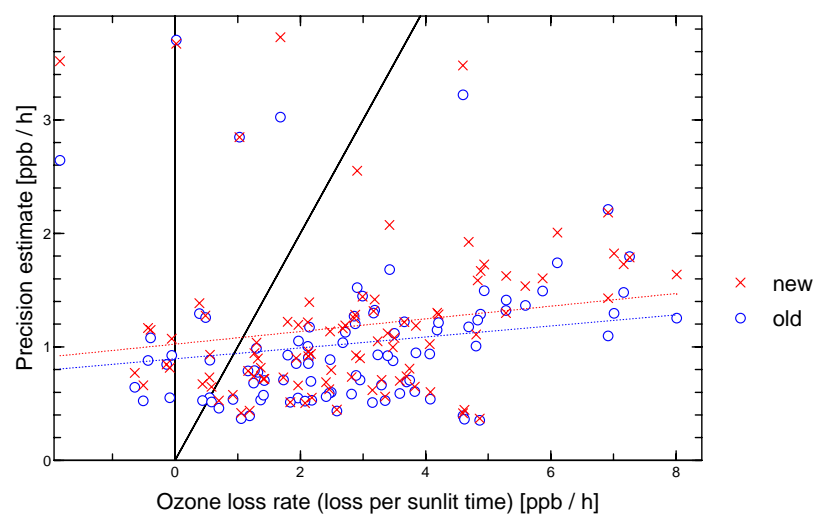

Fig. 7. The new (crosses) and old (circles) estimate of the precision of the ozone loss rate obtained by Match, expressed as one standard deviation, versus the corresponding ozone loss rate. Regression lines for the new and old results have been added (dotted lines). Moreover, the vertical line corresponding to zero ozone loss and the bisecting line (precision estimate of the ozone loss rate $=$ ozone loss rate) have been highlighted. The data represent all match ensembles of the Arctic winters 1994/1995, 1995/1996, 1999/2000, 2002/2003.

concisely, we introduce the following term:

oversampling rate $:=$ average number of matches to which an ozone measurement contributes.

The new error bars have been constructed, in order to account for the multiple use of ozonesonde measurements in several match events. It can be expected that, on average, the new error bars deviate more from the old ones if the oversampling rate increases. This is indeed the case, as can be seen from the regression line added in Fig. 8. The slope of this line is 0.12 , i.e. an increase of the oversampling rate by 1 results in an average increase of the ratio "new error bar/old error bar" by $12 \%$. The regression line almost crosses the point $(1,1)$. This means that the old and new error bars coincide if each sonde measurement is used in only one match event, i.e. if all sondes used in the matches are different from each other. This is also an expected result.

\section{Application to a satellite Match study}

\subsection{Data}

In order to test the formulae of Sect. 7 for larger oversampling rates, i.e. in order to extend Fig. 8 to the right, we consider an additional Match study, which is based on satellite data. Solar occultation instruments, like the Polar Ozone and Aerosol Measurement III (POAM III), perform approximately 14 measurements per day (at a given altitude, in one hemisphere). These measurements are made in latitude bands from $55^{\circ} \mathrm{N}$ to $71^{\circ} \mathrm{N}$ and from $63^{\circ} \mathrm{S}$ to $88^{\circ} \mathrm{S}$. As a consequence the average number of measurements per day

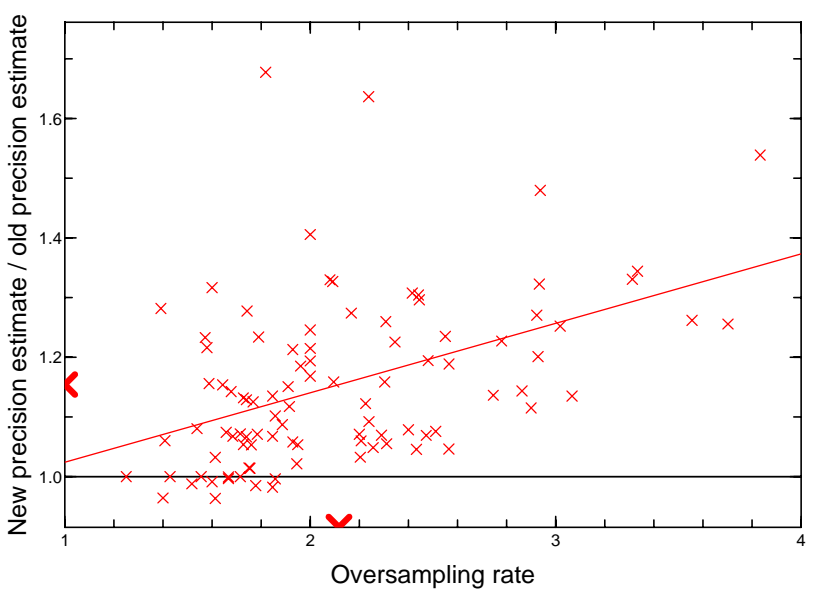

Fig. 8. The ratio "new precision estimate/old precision estimate" versus the oversampling rate ( $=$ the average number of matches to which an ozone measurement contributes). A regression line and the horizontal line corresponding to a ratio of 1 (new error bars = old error bars) have been added. Moreover, the mean values of the abscissae and the ordinates of the data points have been highlighted by triangular arrowheads. The same data as in Fig. 7 have been used.

within the Southern Hemispheric polar vortex is greater than the corresponding number in the Northern Hemispheric vortex. It is also greater than the average number of observations in typical ozonesonde Match campaigns (2-3 per day within the vortex). That is why a relatively large oversampling rate can be expected if the Match technique is applied to satellite data over the Antarctic (cf. Sect. 9.2).

The analysis in the following Sect. 9.2 uses data from an Antarctic Match study based on ozone observations by POAM III in 2003 (potential temperature level $475 \mathrm{~K}$ ). These data correspond to 15 match ensembles, i.e. the calculation of 15 ozone loss rates by the application of a linear regression like in Fig. 1.

\subsection{Comparison of new and old error bars}

For the satellite Match study, the mean oversampling rate is approximately 5 (cf. Fig. 9), i.e. it is significantly larger than for the ozonesonde Match campaigns. An extrapolation of the results of Fig. 8 suggests that this leads to larger ratios of the new errors bars to the old ones. As can be seen in Fig. 9, this is indeed the case. The slope of the regression line added in Fig. 9 is 0.14 , i.e. an increase of the oversampling rate by 1 results in an average increase of the ratio "new error bar/old error bar" by $14 \%$, which is rather similar to the corresponding value for the ozonesonde Match campaigns in Sect. 8.3. For the satellite Match study, the ratio of the new error bars to the old ones varies between 1.1 and 2.8 , the mean value is 1.6. 


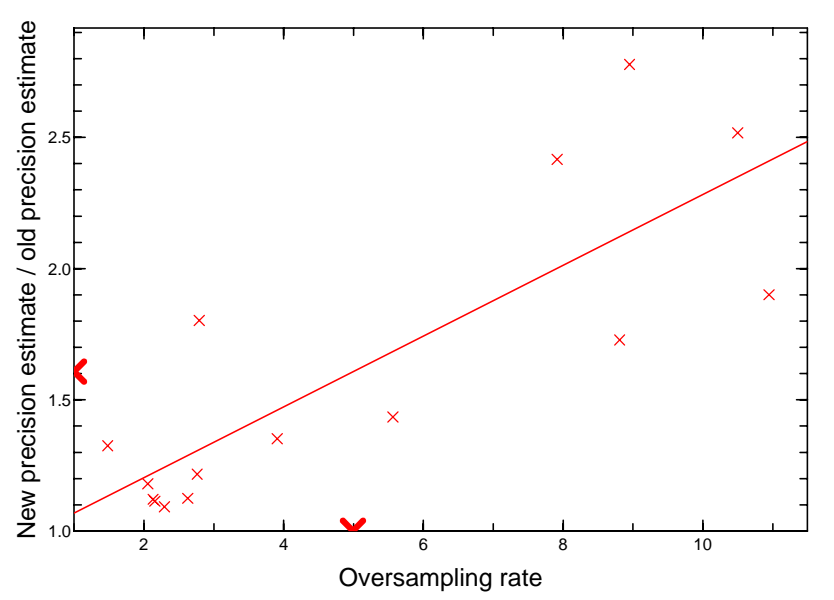

Fig. 9. The ratio "new precision estimate/old precision estimate" versus the oversampling rate, as in Fig. 8. The data represent match ensembles of an Antarctic satellite (POAM III) Match study in 2003.

\section{Conclusions}

A detailed analysis of the random errors of the ozone loss rate calculated by the Match method has been presented. It differs from a standard analysis by taking into account that the same ozonesonde measurement may be used in several matches, so that the ozone mixing ratio differences (second minus first sonde measurement) of these matches become statistically dependent. For four Arctic ozonesonde Match campaigns, this effect leads to changes of the error bars between $-4 \%$ and $+68 \%$. On average, the error bars increase by $15 \%$. This does not change the conclusions about the statistical significance of the ozone loss rates observed. For an Antarctic satellite Match study, the error bars increase by $10 \%$ to $180 \%$, on average by $60 \%$.

Separate estimates for the random errors of the ozonesonde measurements and the random errors arising from the Match method itself show that the errors of these two categories are of similar magnitude. This balance of errors confirms that the criteria for accepting a pair of ozone measurements as a match were suitably selected. The separate estimate of the random measurement errors of the ozonesondes agrees well with the laboratory results of the JOSIE campaigns.

\section{Appendix A: General matrix identities}

Let us assume that $\boldsymbol{e}_{i} \in R^{m}$ denotes the $i$-th unit vector, i.e. its $i$-th element is 1 , all other elements are zero. Then the following identity holds:

$\sum_{i=1}^{m} \boldsymbol{e}_{i} \cdot \boldsymbol{e}_{i}^{T}=\mathbf{I}$
This can be easily proven, because $\boldsymbol{e}_{i} \cdot \boldsymbol{e}_{i}^{T}$ is an $m \times m$ matrix, the $i$-th diagonal element of which is 1 , all other elements are zero.

Let us assume that $\mathbf{A}$ is an $m \times m$ matrix and $\boldsymbol{a}, \boldsymbol{b} \in R^{m}$ are vectors. Then we obtain:

$$
\begin{aligned}
& \sum_{i=1}^{m} \boldsymbol{e}_{i}^{T} \cdot \mathbf{A} \cdot \boldsymbol{a} \cdot \boldsymbol{b}^{T} \cdot \boldsymbol{e}_{i}= \sum_{i=1}^{m} \boldsymbol{b}^{T} \cdot \boldsymbol{e}_{i} \cdot \boldsymbol{e}_{i}^{T} \cdot \mathbf{A} \cdot \boldsymbol{a} \\
& \quad \text { because } \boldsymbol{b}^{T} \cdot \boldsymbol{e}_{i} \text { is a real number, } \\
&= \boldsymbol{b}^{T} \cdot\left(\sum_{i=1}^{m} \boldsymbol{e}_{i} \cdot \boldsymbol{e}_{i}^{T}\right) \cdot \mathbf{A} \cdot \boldsymbol{a} \\
&=\boldsymbol{b}^{T} \cdot \mathbf{A} \cdot \boldsymbol{a} \\
& \quad \text { because of Eq. (63). }
\end{aligned}
$$

\section{Appendix B: Calculation of $E\left(s_{1}\right)$}

The sum $s_{1}$ defined in Eq. (27) can be written in vector notation as

$s_{1}=(\boldsymbol{d}-\hat{r} \cdot \boldsymbol{t})^{T} \cdot(\boldsymbol{d}-\hat{r} \cdot \boldsymbol{t})$.

The term $(\boldsymbol{d}-\hat{r} \cdot \boldsymbol{t})$ occurring in this expression may be transformed as follows:

$$
\begin{aligned}
\boldsymbol{d}-\hat{r} \cdot \boldsymbol{t} & =\boldsymbol{d}-\boldsymbol{t} \cdot \hat{r} \\
& =\boldsymbol{d}-\boldsymbol{t} \cdot \frac{1}{\boldsymbol{t}^{T} \boldsymbol{t}} \cdot \boldsymbol{t}^{T} \boldsymbol{d}, \quad \text { because of Eq. (20), } \\
& =\left(\mathrm{I}-\frac{1}{\boldsymbol{t}^{T} \boldsymbol{t}} \cdot \boldsymbol{t} \cdot \boldsymbol{t}^{T}\right) \cdot \boldsymbol{d} .
\end{aligned}
$$

Let us define the matrix

$\mathbf{J}=\mathbf{I}-\frac{1}{\boldsymbol{t}^{T} \boldsymbol{t}} \cdot \boldsymbol{t} \cdot \boldsymbol{t}^{T}$.

It is symmetric and fulfills the following equations:

$$
\begin{aligned}
\mathbf{J} \cdot \boldsymbol{t} & =\left(\mathbf{I}-\frac{1}{\boldsymbol{t}^{T} \boldsymbol{t}} \cdot \boldsymbol{t} \cdot \boldsymbol{t}^{T}\right) \cdot \boldsymbol{t} \\
& =\boldsymbol{t}-\frac{1}{\boldsymbol{t}^{T} \boldsymbol{t}} \cdot \boldsymbol{t} \cdot \boldsymbol{t}^{T} \boldsymbol{t} \\
& =\boldsymbol{t}-\boldsymbol{t} \\
& =\mathbf{0}, \\
\mathbf{J}^{T} \cdot \mathbf{J} & =\mathbf{J} \cdot \mathbf{J} \\
& =\mathbf{J} \cdot\left(\mathbf{I}-\frac{1}{\boldsymbol{t}^{T} \boldsymbol{t}} \cdot \boldsymbol{t} \cdot \boldsymbol{t}^{T}\right) \\
& =\mathbf{J}-\frac{1}{\boldsymbol{t}^{T} \boldsymbol{t}} \cdot(\mathbf{J} \cdot \boldsymbol{t}) \cdot \boldsymbol{t}^{T} \\
& =\mathbf{J} .
\end{aligned}
$$

Now Eq. (66) can be written as

$$
\begin{aligned}
\boldsymbol{d}-\hat{\boldsymbol{r}} \cdot \boldsymbol{t} & =\mathbf{J} \cdot \boldsymbol{d} \\
& =\mathbf{J} \cdot(\bar{r} \cdot \boldsymbol{t}+\boldsymbol{\epsilon}), \text { because of Eq. (5), } \\
& =\mathbf{J} \cdot \boldsymbol{\epsilon}, \text { because of Eq. (68). }
\end{aligned}
$$


Then the expected value of $s_{1}$ defined in Eq. (65) can be calculated:

$$
\begin{aligned}
& \mathrm{E}\left(s_{1}\right)=\mathrm{E}\left((\boldsymbol{d}-\hat{r} \cdot \boldsymbol{t})^{T} \cdot(\boldsymbol{d}-\hat{r} \cdot \boldsymbol{t})\right) \\
& =\mathrm{E}\left(\boldsymbol{\epsilon}^{T} \cdot \mathbf{J}^{T} \cdot \mathbf{J} \cdot \boldsymbol{\epsilon}\right) \text {, because of Eq. (70), } \\
& =\mathrm{E}\left(\boldsymbol{\epsilon}^{T} \cdot \mathbf{J} \cdot \boldsymbol{\epsilon}\right) \text {, because of Eq. (69), } \\
& =\mathrm{E}\left\{\boldsymbol{\epsilon}^{T} \cdot\left(\mathbf{I}-\frac{1}{\boldsymbol{t}^{T} \boldsymbol{t}} \cdot \boldsymbol{t} \cdot \boldsymbol{t}^{T}\right) \cdot \boldsymbol{\epsilon}\right\} \\
& =\mathrm{E}\left(\boldsymbol{\epsilon}^{T} \boldsymbol{\epsilon}\right)-\frac{1}{\boldsymbol{t}^{T} \boldsymbol{t}} \cdot \mathrm{E}\left(\boldsymbol{\epsilon}^{T} \cdot \boldsymbol{t} \cdot \boldsymbol{t}^{T} \cdot \boldsymbol{\epsilon}\right) \\
& =\mathrm{E}\left(\sum_{i=1}^{m} \epsilon_{i}^{2}\right)-\frac{1}{\boldsymbol{t}^{T} \boldsymbol{t}} \cdot \mathrm{E}\left(\boldsymbol{t}^{T} \cdot \boldsymbol{\epsilon} \cdot \boldsymbol{\epsilon}^{T} \cdot \boldsymbol{t}\right) \\
& \text { because } \boldsymbol{\epsilon}^{T} \cdot \boldsymbol{t}=\boldsymbol{t}^{T} \cdot \boldsymbol{\epsilon}, \\
& =\left(\sum_{i=1}^{m} \mathrm{E}\left(\epsilon_{i}^{2}\right)\right)-\frac{1}{\boldsymbol{t}^{T} \boldsymbol{t}} \cdot \boldsymbol{t}^{T} \cdot \mathrm{E}\left(\boldsymbol{\epsilon} \cdot \boldsymbol{\epsilon}^{T}\right) \cdot \boldsymbol{t} \\
& =\left(\sum_{i=1}^{m} \operatorname{Var}\left(\epsilon_{i}\right)\right)-\frac{1}{\boldsymbol{t}^{T} \boldsymbol{t}} \cdot \boldsymbol{t}^{T} \cdot \operatorname{Cov}(\boldsymbol{\epsilon}) \cdot \boldsymbol{t}, \\
& \text { because of } \mathrm{E}(\boldsymbol{\epsilon})=\mathbf{0} \text {, } \\
& =\left(\sum_{i=1}^{m} \sigma^{2}\right)-\frac{1}{\boldsymbol{t}^{T} \boldsymbol{t}} \cdot \boldsymbol{t}^{T} \cdot\left(\sigma^{2} \cdot \mathbf{I}+\sigma_{\delta}^{2} \cdot \boldsymbol{\Omega}\right) \cdot \boldsymbol{t},
\end{aligned}
$$

because of Eq. (18),

$$
\begin{aligned}
& =m \cdot \sigma^{2}-\frac{\boldsymbol{t}^{T} \boldsymbol{t}}{\boldsymbol{t}^{T} \boldsymbol{t}} \cdot \sigma^{2}-\frac{\boldsymbol{t}^{T} \boldsymbol{\Omega} \boldsymbol{t}}{\boldsymbol{t}^{T} \boldsymbol{t}} \cdot \sigma_{\delta}^{2} \\
& =(m-1) \cdot \sigma^{2}-\frac{\boldsymbol{t}^{T} \boldsymbol{\Omega} \boldsymbol{t}}{\boldsymbol{t}^{T} \boldsymbol{t}} \cdot \sigma_{\delta}^{2} .
\end{aligned}
$$

Together with the definition of $\omega$ in Eq. (23) we thus obtain

$$
\mathrm{E}\left(s_{1}\right)=(m-1) \cdot \sigma^{2}-\omega \cdot \sigma_{\delta}^{2} .
$$

\section{Appendix C: Calculation of $E\left(s_{2}\right)$}

The sum $s_{2}$ defined in Eq. (39) can be transformed to vector notation as follows:

$$
\begin{aligned}
s_{2} & =\sum_{i=1}^{m} \sum_{j=1}^{m} \omega_{i j} \cdot\left(d_{i}-\hat{r} \cdot t_{i}\right) \cdot\left(d_{j}-\hat{r} \cdot t_{j}\right) \\
& =\sum_{i=1}^{m} \sum_{j=1}^{m} \boldsymbol{e}_{i}^{T} \boldsymbol{\Omega} \boldsymbol{e}_{j} \cdot \boldsymbol{e}_{i}^{T}(\boldsymbol{d}-\hat{r} \cdot \boldsymbol{t}) \cdot \boldsymbol{e}_{j}^{T}(\boldsymbol{d}-\hat{r} \cdot \boldsymbol{t}) \\
& =\sum_{i=1}^{m} \sum_{j=1}^{m} \boldsymbol{e}_{i}^{T} \boldsymbol{\Omega} \boldsymbol{e}_{j} \cdot \boldsymbol{e}_{j}^{T}(\boldsymbol{d}-\hat{r} \cdot \boldsymbol{t}) \cdot(\boldsymbol{d}-\hat{r} \cdot \boldsymbol{t})^{T} \boldsymbol{e}_{i} \\
& =\sum_{i=1}^{m} \boldsymbol{e}_{i}^{T} \boldsymbol{\Omega} \cdot\left(\sum_{j=1}^{m} \boldsymbol{e}_{j} \cdot \boldsymbol{e}_{j}^{T}\right) \cdot(\boldsymbol{d}-\hat{r} \cdot \boldsymbol{t}) \cdot(\boldsymbol{d}-\hat{r} \cdot \boldsymbol{t})^{T} \boldsymbol{e}_{i}
\end{aligned}
$$

$$
=\sum_{i=1}^{m} \boldsymbol{e}_{i}^{T} \boldsymbol{\Omega} \cdot(\boldsymbol{d}-\hat{r} \cdot \boldsymbol{t}) \cdot(\boldsymbol{d}-\hat{r} \cdot \boldsymbol{t})^{T} \boldsymbol{e}_{i},
$$

$$
\text { because of Eq. (63). }
$$

Thus we obtain:

$$
\begin{aligned}
\mathrm{E}\left(s_{2}\right) & =\sum_{i=1}^{m} \boldsymbol{e}_{i}^{T} \boldsymbol{\Omega} \cdot \mathrm{E}\left\{(\boldsymbol{d}-\hat{r} \cdot \boldsymbol{t}) \cdot(\boldsymbol{d}-\hat{r} \cdot \boldsymbol{t})^{T}\right\} \cdot \boldsymbol{e}_{i} \\
& =\sum_{i=1}^{m} \boldsymbol{e}_{i}^{T} \cdot \boldsymbol{\Omega} \cdot \mathrm{E}\left(\mathbf{J} \cdot \boldsymbol{\epsilon} \cdot \boldsymbol{\epsilon}^{T} \cdot \mathbf{J}^{T}\right) \cdot \boldsymbol{e}_{i}
\end{aligned}
$$

because of Eq. (70),

$$
=\sum_{i=1}^{m} \boldsymbol{e}_{i}^{T} \cdot \boldsymbol{\Omega} \cdot \mathbf{J} \cdot \operatorname{Cov}(\boldsymbol{\epsilon}) \cdot \mathbf{J} \cdot \boldsymbol{e}_{i},
$$

because of $\mathrm{E}(\boldsymbol{\epsilon})=\mathbf{0}$ and the symmetry of $\mathbf{J}$,

$$
=\sum_{i=1}^{m} \boldsymbol{e}_{i}^{T} \cdot \boldsymbol{\Omega} \cdot \mathbf{J} \cdot\left(\sigma^{2} \cdot \mathbf{I}+\sigma_{\delta}^{2} \cdot \boldsymbol{\Omega}\right) \cdot \mathbf{J} \cdot \boldsymbol{e}_{i},
$$

because of Eq. (18),

$$
\begin{aligned}
= & \left(\sum_{i=1}^{m} \boldsymbol{e}_{i}^{T} \cdot \boldsymbol{\Omega} \cdot \mathbf{J} \cdot \mathbf{J} \cdot \boldsymbol{e}_{i}\right) \cdot \sigma^{2} \\
& +\left(\sum_{i=1}^{m} \boldsymbol{e}_{i}^{T} \cdot \boldsymbol{\Omega} \cdot \mathbf{J} \cdot \boldsymbol{\Omega} \cdot \mathbf{J} \cdot \boldsymbol{e}_{i}\right) \cdot \sigma_{\delta}^{2} .
\end{aligned}
$$

The expressions in front of $\sigma^{2}$ and $\sigma_{\delta}^{2}$ are evaluated separately:

$$
\begin{aligned}
& \sum_{i=1}^{m} \boldsymbol{e}_{i}^{T} \cdot \boldsymbol{\Omega} \cdot \mathbf{J} \cdot \mathbf{J} \cdot \boldsymbol{e}_{i} \\
= & \sum_{i=1}^{m} \boldsymbol{e}_{i}^{T} \cdot \boldsymbol{\Omega} \cdot \mathbf{J} \cdot \boldsymbol{e}_{i}, \text { because of Eq. (69), } \\
= & \sum_{i=1}^{m} \boldsymbol{e}_{i}^{T} \cdot \boldsymbol{\Omega} \cdot\left(\mathbf{I}-\frac{1}{\boldsymbol{t}^{T} \boldsymbol{t}} \cdot \boldsymbol{t} \cdot \boldsymbol{t}^{T}\right) \cdot \boldsymbol{e}_{i}
\end{aligned}
$$

because of the definition of $\mathbf{J}$ in Eq. (67),

$=\sum_{i=1}^{m} \boldsymbol{e}_{i}^{T} \cdot \boldsymbol{\Omega} \cdot \boldsymbol{e}_{i}-\frac{1}{\boldsymbol{t}^{T} \boldsymbol{t}} \cdot \sum_{i=1}^{m} \boldsymbol{e}_{i}^{T} \cdot \boldsymbol{\Omega} \cdot \boldsymbol{t} \cdot \boldsymbol{t}^{T} \cdot \boldsymbol{e}_{i}$

$=\sum_{i=1}^{m} \omega_{i i}-\frac{1}{\boldsymbol{t}^{T} \boldsymbol{t}} \cdot \boldsymbol{t}^{T} \cdot \boldsymbol{\Omega} \cdot \boldsymbol{t}$, because of Eq. (64),

$=-\frac{1}{t^{T} t} \cdot t^{T} \cdot \boldsymbol{\Omega} \cdot \boldsymbol{t}$,

because all diagonal elements of $\boldsymbol{\Omega}$ are zero,

cf. Eq. (15),

$=-\omega$, because of the definition of $\omega$ in Eq. (23).

The expression in front of $\sigma_{\delta}^{2}$ in Eq. (73) is

$$
\sum_{i=1}^{m} \boldsymbol{e}_{i}^{T} \cdot \boldsymbol{\Omega} \cdot \mathbf{J} \cdot \boldsymbol{\Omega} \cdot \mathbf{J} \cdot \boldsymbol{e}_{i}
$$




$$
\begin{aligned}
=\sum_{i=1}^{m} \boldsymbol{e}_{i}^{T} \cdot \boldsymbol{\Omega} \cdot\left(\mathbf{I}-\frac{1}{\boldsymbol{t}^{T} \boldsymbol{t}} \cdot \boldsymbol{t} \cdot \boldsymbol{t}^{T}\right) \\
\cdot \boldsymbol{\Omega} \cdot\left(\mathbf{I}-\frac{1}{\boldsymbol{t}^{T} \boldsymbol{t}} \cdot \boldsymbol{t} \cdot \boldsymbol{t}^{T}\right) \cdot \boldsymbol{e}_{i},
\end{aligned}
$$

because of the definition of $\mathbf{J}$ in Eq. (67),

$$
\begin{aligned}
= & \sum_{i=1}^{m} \boldsymbol{e}_{i}^{T} \cdot \boldsymbol{\Omega} \cdot \boldsymbol{\Omega} \cdot \boldsymbol{e}_{i}-\frac{1}{\boldsymbol{t}^{T} \boldsymbol{t}} \cdot \sum_{i=1}^{m} \boldsymbol{e}_{i}^{T} \cdot \boldsymbol{\Omega} \cdot \boldsymbol{\Omega} \cdot \boldsymbol{t} \cdot \boldsymbol{t}^{T} \cdot \boldsymbol{e}_{i} \\
& -\frac{1}{\boldsymbol{t}^{T} \boldsymbol{t}} \cdot \sum_{i=1}^{m} \boldsymbol{e}_{i}^{T} \cdot \boldsymbol{\Omega} \cdot \boldsymbol{t} \cdot \boldsymbol{t}^{T} \cdot \boldsymbol{\Omega} \cdot \boldsymbol{e}_{i} \\
& +\frac{1}{\left(\boldsymbol{t}^{T} \boldsymbol{t}\right)^{2}} \cdot \sum_{i=1}^{m} \boldsymbol{e}_{i}^{T} \cdot \boldsymbol{\Omega} \cdot \boldsymbol{t} \cdot \boldsymbol{t}^{T} \cdot \boldsymbol{\Omega} \cdot \boldsymbol{t} \cdot \boldsymbol{t}^{T} \cdot \boldsymbol{e}_{i}
\end{aligned}
$$

We are going to evaluate the four sums occurring in Eq. (75) separately:

$$
\begin{aligned}
& \sum_{i=1}^{m} \boldsymbol{e}_{i}^{T} \cdot \boldsymbol{\Omega} \cdot \boldsymbol{\Omega} \cdot \boldsymbol{e}_{i}=\sum_{j=1}^{m}\left(\boldsymbol{\Omega} \cdot \boldsymbol{e}_{j}\right)^{T} \cdot \boldsymbol{\Omega} \cdot \boldsymbol{e}_{j} \\
& \text { because } \boldsymbol{\Omega} \text { is symmetric, } \\
&=\sum_{j=1}^{m}\left(\begin{array}{c}
\omega_{1 j} \\
\cdots \\
\omega_{m j}
\end{array}\right) \cdot\left(\begin{array}{c}
\omega_{1 j} \\
\cdots \\
\omega_{m j}
\end{array}\right) \\
&=\sum_{i=1}^{m} \sum_{j=1}^{m} \omega_{i j}^{2} .
\end{aligned}
$$

$\sum_{i=1}^{m} \boldsymbol{e}_{i}^{T} \cdot \boldsymbol{\Omega} \cdot \boldsymbol{\Omega} \cdot \boldsymbol{t} \cdot \boldsymbol{t}^{T} \cdot \boldsymbol{e}_{i}=\boldsymbol{t}^{T} \cdot \boldsymbol{\Omega}^{2} \cdot \boldsymbol{t}$

because of Eq. (64).

$$
\begin{aligned}
\sum_{i=1}^{m} \boldsymbol{e}_{i}^{T} \cdot \boldsymbol{\Omega} \cdot \boldsymbol{t} \cdot \boldsymbol{t}^{T} \cdot \boldsymbol{\Omega} \cdot \boldsymbol{e}_{i} & =\boldsymbol{t}^{T} \cdot \boldsymbol{\Omega} \cdot \boldsymbol{\Omega} \cdot \boldsymbol{t} \\
& \text { because of Eq. (64) } \\
& \text { with } \boldsymbol{b}^{T} \hat{=} \boldsymbol{t}^{T} \cdot \boldsymbol{\Omega}, \\
& =\boldsymbol{t}^{T} \cdot \boldsymbol{\Omega}^{2} \cdot \boldsymbol{t} .
\end{aligned}
$$

$$
\sum_{i=1}^{m} \boldsymbol{e}_{i}^{T} \cdot \boldsymbol{\Omega} \cdot \boldsymbol{t} \cdot \boldsymbol{t}^{T} \cdot \boldsymbol{\Omega} \cdot \boldsymbol{t} \cdot \boldsymbol{t}^{T} \cdot \boldsymbol{e}_{i}=\boldsymbol{t}^{T} \cdot \boldsymbol{\Omega} \cdot \boldsymbol{t} \cdot \boldsymbol{t}^{T} \cdot \boldsymbol{\Omega} \cdot \boldsymbol{t}
$$
because of Eq. (64),

$$
=\left(\boldsymbol{t}^{T} \cdot \boldsymbol{\Omega} \cdot \boldsymbol{t}\right)^{2}
$$

By substituting the expressions of Eqs. (76)-(79) in Eq. (75) we obtain:

$$
\begin{aligned}
& \sum_{i=1}^{m} \boldsymbol{e}_{i}^{T} \cdot \boldsymbol{\Omega} \cdot \mathbf{J} \cdot \boldsymbol{\Omega} \cdot \mathbf{J} \cdot \boldsymbol{e}_{i} \\
= & \sum_{i=1}^{m} \sum_{j=1}^{m} \omega_{i j}^{2}-\frac{1}{\boldsymbol{t}^{T} \boldsymbol{t}} \cdot \boldsymbol{t}^{T} \cdot \boldsymbol{\Omega}^{2} \cdot \boldsymbol{t}-\frac{1}{\boldsymbol{t}^{T} \boldsymbol{t}} \cdot \boldsymbol{t}^{T} \cdot \boldsymbol{\Omega}^{2} \cdot \boldsymbol{t} \\
& +\frac{1}{\left(\boldsymbol{t}^{T} \boldsymbol{t}\right)^{2}} \cdot\left(\boldsymbol{t}^{T} \cdot \boldsymbol{\Omega} \cdot \boldsymbol{t}\right)^{2}
\end{aligned}
$$

$$
\begin{aligned}
= & \sum_{i=1}^{m} \sum_{j=1}^{m} \omega_{i j}^{2}-2 \cdot \frac{\boldsymbol{t}^{T} \cdot \boldsymbol{\Omega}^{2} \cdot \boldsymbol{t}}{\boldsymbol{t}^{T} \boldsymbol{t}}+\left(\frac{\boldsymbol{t}^{T} \cdot \boldsymbol{\Omega} \cdot \boldsymbol{t}}{\boldsymbol{t}^{T} \boldsymbol{t}}\right)^{2} \\
= & \sum_{\substack{i=1 \\
\text { because } \boldsymbol{\Omega} \text { is symmetric, } \\
\quad}}^{m} \omega_{i j}^{2}-2 \cdot \frac{(\boldsymbol{\Omega} \cdot \boldsymbol{t})^{T} \cdot \boldsymbol{\Omega} \cdot \boldsymbol{t}}{\boldsymbol{t}^{T} \boldsymbol{t}}+\left(\frac{\boldsymbol{t}^{T} \cdot \boldsymbol{\Omega} \cdot \boldsymbol{t}}{\boldsymbol{t}^{T} \boldsymbol{t}}\right)^{2}, \\
= & \omega_{1}-2 \cdot \omega_{2}+\omega^{2},
\end{aligned}
$$$$
\text { because of the definition of } \omega_{1}, \omega_{2} \text {, and } \omega \text { in }
$$$$
\text { Eqs. (40), (41), (23). }
$$

By substituting the expressions of Eqs. (74) and (80) in Eq. (73) we obtain:

$\mathrm{E}\left(s_{2}\right)=-\omega \cdot \sigma^{2}+\left(\omega_{1}-2 \cdot \omega_{2}+\omega^{2}\right) \cdot \sigma_{\delta}^{2}$.

Acknowledgement. We thank K. Hoppel (Naval Research Laboratory, Washington) for providing the POAM III data on which the analysis in Sect. 9 is based. This work was partly supported by the QUOBI project of the EC DG Research under the contract EVK2-2001-00129.

Edited by: W. Ward

\section{References}

Grooß, J.-U. and Müller, R.: The impact of mid-latitude intrusions into the polar vortex on ozone loss estimates, Atmos. Chem. Phys., 3, 395-402, 2003,

SRef-ID: 1680-7324/acp/2003-3-395.

Morris, G. A., Bojkov, B. R., Lait, L. R., and Schoeberl, M. R.: A review of the Match technique as applied to AASE-2/EASOE and SOLVE/THESEO 2000, Atmos. Chem. Phys., 5, 25712592, 2005,

SRef-ID: 1680-7324/acp/2005-5-2571.

Rex, M., Harris, N. R. P., von der Gathen, P., et al.: Prolonged stratospheric ozone loss in the 1995-96 Arctic winter, Nature, 389, 835-838, 1997.

Rex, M., von der Gathen, P., Harris, N. R. P., et al.: In situ measurements of stratospheric ozone depletion rates in the Arctic winter 1991/1992: A Lagrangian approach, J. Geophys. Res., 103, 5843-5853, 1998

Rex, M., von der Gathen, P., Braathen, G. O., et al.: Chemical ozone loss in the Arctic winter 1994/95 as determined by the Match technique, J. Atmos. Chem., 32, 35-59, 1999.

Rex, M., Salawitch, R. J., Harris, N. R. P., et al.: Chemical depletion of Arctic ozone in winter 1999/2000, J. Geophys. Res., 107, 8276, doi:10.1029/2001JD000533, 2002.

Sasano, Y., Terao, Y., Tanaka, L., Yasunari, T., Kanzawa, H., Nakajima, H., Yokota, T., Nakane, H., Hayashida, S., and Saitoh, N.: ILAS observations of chemical ozone loss in the Arctic vortex during early spring 1997, Geophys. Res. Lett., 27, 213-216, 2000.

Smit, H. G. J. and Straeter, W.: JOSIE-1998, Performance of ECC Ozone Sondes of SPC-6A and ENSCI-Z Type, WMO Global Atmosphere Watch report series, No. 157 (Technical Document No. 1218), World Meteorological Organization, Geneva, 2004a. 
Smit, H. G. J. and Straeter, W.: JOSIE-2000, Jülich Ozone Sonde Intercomparison Experiment 2000, The 2000 WMO international intercomparison of operating procedures for ECCozone sondes at the environmental simulation facility at Jülich, WMO Global Atmosphere Watch report series, No. 158 (Technical Document No. 1225), World Meteorological Organization, Geneva, 2004b.

Streibel, M., Rex, M., von der Gathen, P., et al.: Chemical ozone loss in the Arctic winter 2002/2003 determined with Match, Atmos. Chem. Phys. Discuss., 5, 4311-4333, 2005,

SRef-ID: 1680-7375/acpd/2005-5-4311.
Terao, Y., Sasano, Y., Nakajima, H., Tanaka, L., and Yasunari, T.: Stratospheric ozone loss in the 1996/1997 Arctic winter: Evaluation based on multiple trajectory analysis for doublesounded air parcels by ILAS, J. Geophys. Res., 107, 8210, doi:10.1029/2001JD000615, 2002.

von der Gathen, P., Rex, M., Harris, N. R. P., et al.: Observational evidence for chemical ozone depletion over the Arctic in winter 1991-92, Nature, 375, 131-134, 1995. 